\title{
Myocardial perfusion scintigraphy in the UK: insights from the British Nuclear Cardiology Society Survey 2000
}

\author{
A D Kelion, C Anagnostopoulos, M Harbinson, S R Underwood, M Metcalfe, for the British \\ Nuclear Cardiology Society
}

Heart 2005;91(Suppl IV):iv2-iv5. doi: 10.1136/hrt.2004.049759

See end of article for authors' affiliations

Correspondence to: Dr Andrew D Kelion, Nuclear Medicine Department, Harefield Hospital, Hill End Road, Harefield, Middlesex UB9 6JH, UK; A.Kelion@rbh. nthames.nhs.uk

\begin{abstract}
Background: The National Institute for Health and Clinical Excellence (NICE) has recently published a very positive technology appraisal of myocardial perfusion scintigraphy (MPS). This has important implications for service provision within the National Health Service, and an accurate knowledge of the current level of MPS activity is necessary.

Methods: A postal questionnaire was sent to 207 nuclear medicine departments in the UK, requesting information about nuclear cardiology facilities, activity, and practice. Non-responding departments were sent a second questionnaire, followed where necessary by a telephone call.

Results: A response rate of $61 \%$ was achieved; $52 \%$ of departments performed MPS, and these tended to have more gamma cameras than those which did not (median (25th-75th centile) 2.0, 1.5-2.5 v 1.0, $0.5-1.5 ; p=0.02)$. The median number of studies performed was 256 (144-460). The estimated rate of MPS in the UK for the year 2000 was 1200 per million population. The median (25th-75th centile) waiting time for MPS was 16 (9-24) weeks. Pharmacological stress was used in 77\% of studies, and a technetium$99 \mathrm{~m}$ based radiopharmaceutical in 60\% (two day protocol in 75\%). Tomographic rather than planar imaging was performed in $88 \%$ of studies, of which $22 \%$ were ECG gated. A cardiologist was involved in reporting in $35 \%$ of studies.

Conclusions: MPS activity in the UK remains low, and it tends to be provided as a low volume service with unacceptably long waiting times and a lack of involvement by cardiologists. The recent NICE appraisal may provide an impetus for further resourcing and development.
\end{abstract}

M yocardial perfusion scintigraphy (MPS) is a noninvasive investigation which provides robust, cost effective, diagnostic and prognostic information in patients with known or suspected coronary disease. ${ }^{1}$ The British Cardiac Society (BCS) has recommended the routine first line use of MPS in the investigation of chest pain when the exercise ECG is likely to be unreliable, as in patients unable to exercise, those with an abnormal resting ECG, or women. ${ }^{2}$ Such guidance was not reflected in the National Service Framework (NSF) for coronary heart disease document published in 2000, and anecdotal reports suggest that this may have led to an inefficient use of resources. ${ }^{3}$ For example, the rate of normal coronary angiograms was as high as $56 \%$ among women referred from one rapid access chest pain clinic. ${ }^{4}$

The National Institute for Health and Clinical Excellence (NICE) has recently completed a technology appraisal of MPS, and its findings have been positive. ${ }^{5}$ MPS is recommended as an initial diagnostic tool in patients for whom the exercise ECG is likely to be unreliable (as in the BCS guidelines), as well as in diabetics and lower risk patients. Moreover, it should form part of the investigational strategy in patients with known coronary disease who remain symptomatic following myocardial infarction or revascularisation. These recommendations may have important implications for service provision within the National Health Service, and an accurate knowledge of the current level of MPS activity is therefore mandatory. The British Nuclear Cardiology Society (BNCS) has conducted regular surveys of MPS practice in the UK. ${ }^{6-8}$ We report the results of the fourth and most recent survey, for the year 2000.

\section{MATERIALS AND METHODS}

In the spring of 2001, a postal questionnaire was sent to each of 207 nuclear medicine departments in the UK, identified from lists supplied by industry and the BNCS membership database. The questionnaire requested information about nuclear cardiology facilities, activity and practice. Nonresponding departments were sent a second questionnaire, followed where necessary by a telephone call.

The calculation of accurate national rates of MPS from the survey is complicated by the incomplete response rate to any questionnaire, and an assumption must be made about activity in non-responding centres. Telephone follow up identified departments which performed no MPS, and the majority of departments in the non-responding group therefore performed MPS but were unable or unwilling to provide data. National figures were therefore calculated assuming that non-responding centres performed the same mean number of MPSs per centre as responding cardiac centres. The UK population in 2000 was taken to be 58 million.

Continuous variables are expressed as mean (SD) or median (25th-75th centile), as appropriate. Continuous variables were compared using an unpaired $t$ test, or a Mann-Whitney $U$ test when there was significant deviation from the normal distribution. Categorical variables were compared using a $\chi^{2}$ test. A probability value of $p<0.05$ was taken to denote significance.

\section{RESULTS}

\section{General information about departments}

Useful information was obtained from 127 (61\%) departments. One hundred and nine were in England, eight in

\footnotetext{
Abbreviations: BCS, British Cardiac Society; BNCS, British Nuclear Cardiology Society; DGH, district general hospital; MPS, myocardial perfusion scintigraphy; NICE, National Institute for Health and Clinical Excellence; NSF, National Service Framework; SPECT, single photon emission computed tomography
} 
Scotland, five in Wales, two in Northern Ireland, and one in the Isle of Man; 73\% were in district general hospitals (DGHs), 20\% were in tertiary cardiac centres, and the remaining $7 \%$ were in various private and specialist hospitals. Only three departments were dedicated nuclear cardiology facilities, while the remainder offered a general nuclear medicine service. Fifty four per cent of departments had one gamma camera only, of which $86 \%$ had single photon emission computed tomographic (SPECT) capability; $32 \%$ had two cameras, with SPECT capability on at least one in $94 \%$ of cases; $14 \%$ had three or more cameras, with SPECT capability on at least one in all cases. Five departments had one or more dedicated cardiac cameras. The median (25th75 th centile) number of all nuclear medicine studies performed in the year 2000 was 2500 (1800-3500).

\section{MPS activity}

Sixty six $(52 \%)$ of responding departments performed MPS: $58 \%$ of DGHs and $84 \%$ of tertiary cardiac centres. Compared with departments performing no MPS, MPS centres had more gamma cameras (median, 25th-75th centile: 2.0, 1.5$2.5 v 1.0,0.5-1.5 ; \mathrm{p}=0.02)$ and performed more overall nuclear medicine studies (mean (SD) 3619 (2590) v 2344 (1090); $p=0.007)$. The estimated rate of MPS in the UK for the year 2000 was 1200 per million population (fig 1). For departments performing MPS, the median (25th-75th centile) number of studies was 256 (144-460) (fig 2). The busiest quartile of centres accounted for $68 \%$ of national activity. These high volume MPS centres possessed 38\% of the gamma cameras and performed $41 \%$ of overall nuclear medicine studies. Compared with MPS, 2.9 times as many exercise ECGs and 1.9 times as many coronary angiograms were performed in those hospitals which provided data.

\section{MPS referrals}

The median (25th-75th centile) waiting time for MPS was 16 (9-24) weeks (similar across activity quartiles); 92\% of MPS studies were performed on outpatients. External referrals accounted for $47 \%$ of the workload of the busiest quartile of centres, but only $16 \%$ of the workload of the less busy centres $(p=0.005 ; 37 \%$ overall $)$. Forty one per cent of referrals were from interventional cardiologists, 37\% from non-interventional cardiologists, $17 \%$ from general physicians, and 5\% from surgeons (similar across activity quartiles). Sixty two

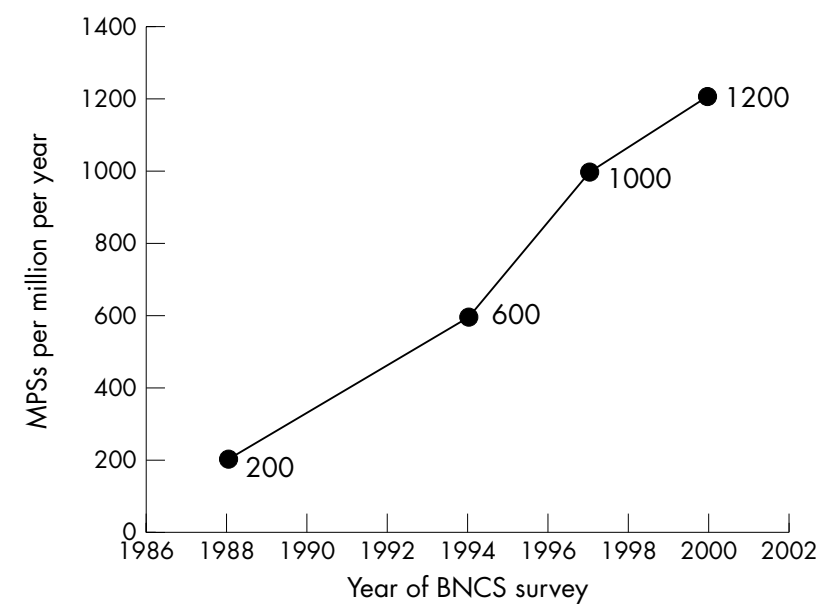

Figure 1 Number of myocardial perfusion scintigraphy (MPS) studies per million population per year from the four British Nuclear Cardiology Society (BNCS) surveys. ${ }^{6-8}$ Values were calculated assuming that nonresponding departments performed the same mean number of MPSs per centre as responding centres which performed MPS. The UK population was taken to be 58 million.

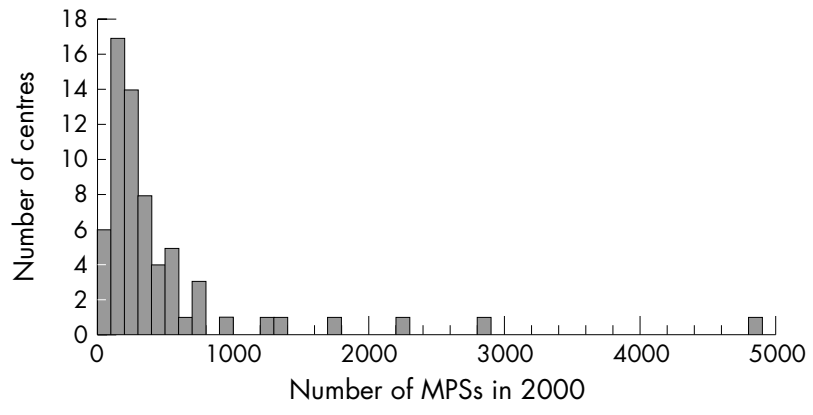

Figure 2 Frequency histogram of numbers of MPS performing centres according to annual number of studies performed. Mean (SD) 479 (739), median (25th-75th centile) 256 (144-460).

per cent of referrals were for investigation of chest pain, $16 \%$ were for post-infarct risk assessment, 17\% were to guide management following coronary angiography or revascularisation, and 5\% were for risk stratification before non-cardiac surgery (similar across activity quartiles).

\section{MPS stress}

Departments in the busiest activity quartile used fewer members of staff to perform stress than less busy departments (median, 25th-75th centile: 2.0, 2.0-2.0 v 3.0, 2.5-3.5; $p=0.004 ; 2.0,1.5-2.5$ overall). Stress tests were under the direct supervision of a doctor in $77 \%$ of departments, a cardiac technician in $12 \%$, a nurse in $8 \%$, and a radiographer in $3 \%$. Dynamic exercise stress was used in $23 \%$ of studies (treadmill 50\%, ergometer 50\%), vasodilator stress in 66\%, and dobutamine in $11 \%$ (fig 3). Adenosine rather than dipyridamole was used for vasodilator stress more often in departments in the busiest activity quartile compared with the less busy quartiles ( $80 \%$ v 57\%; p $<0.0001 ; 72 \%$ overall). Vasodilator stress was more likely to be combined with submaximal exercise in the busiest centres compared with the less busy (59\% v 35\%; p $<0.0001 ; 51 \%$ overall). The majority of centres used mainly $(76 \%)$ or exclusively $(52 \%)$ pharmacological stress; the busiest quartile of centres tended to use at least some dynamic exercise compared with the less busy $(62 \% \vee 44 \% ; \mathrm{p}=\mathrm{NS})$.

\section{MPS radiopharmaceuticals}

The radiopharmaceutical used in MPS was thallium-201 in $40 \%$ of studies, technetium-99m-tetrofosmin in $46 \%$, and technetium-99m-sestamibi in $14 \%$ (fig 3). In the busiest quartile of departments, thallium was used in $50 \%$ of studies, compared with only $18 \%$ of studies in less busy centres $(p<0.0001)$. When a technetium-99m based radiopharmaceutical was used, a one day rather than a two day protocol was chosen in $25 \%$ of cases, of which $81 \%$ were stress-rest rather than rest-stress; $78 \%$ of departments used a technetium-99m based agent exclusively $(67 \%$ of centres in the busiest quartile, compared with $82 \%$ of less busy centres; $\mathrm{p}=\mathrm{NS})$.

\section{MPS imaging}

MPS image acquisition was performed by a nuclear medicine technician in $55 \%$ of centres, a radiographer in $35 \%$, a nuclear medicine technician or radiographer in $8 \%$, and a cardiac technician in $2 \%$. SPECT rather than planar imaging was performed in $88 \%$ of studies. Twenty eight per cent of centres performed gated SPECT ( $57 \%$ of centres in the upper activity quartile compared with $19 \%$ of quieter centres; $p=0.01$ ), and $22 \%$ of SPECT MPS studies overall involved gating. Nineteen per cent of centres used attenuation correction (7\% of centres in the upper activity quartile compared with $23 \%$ of 

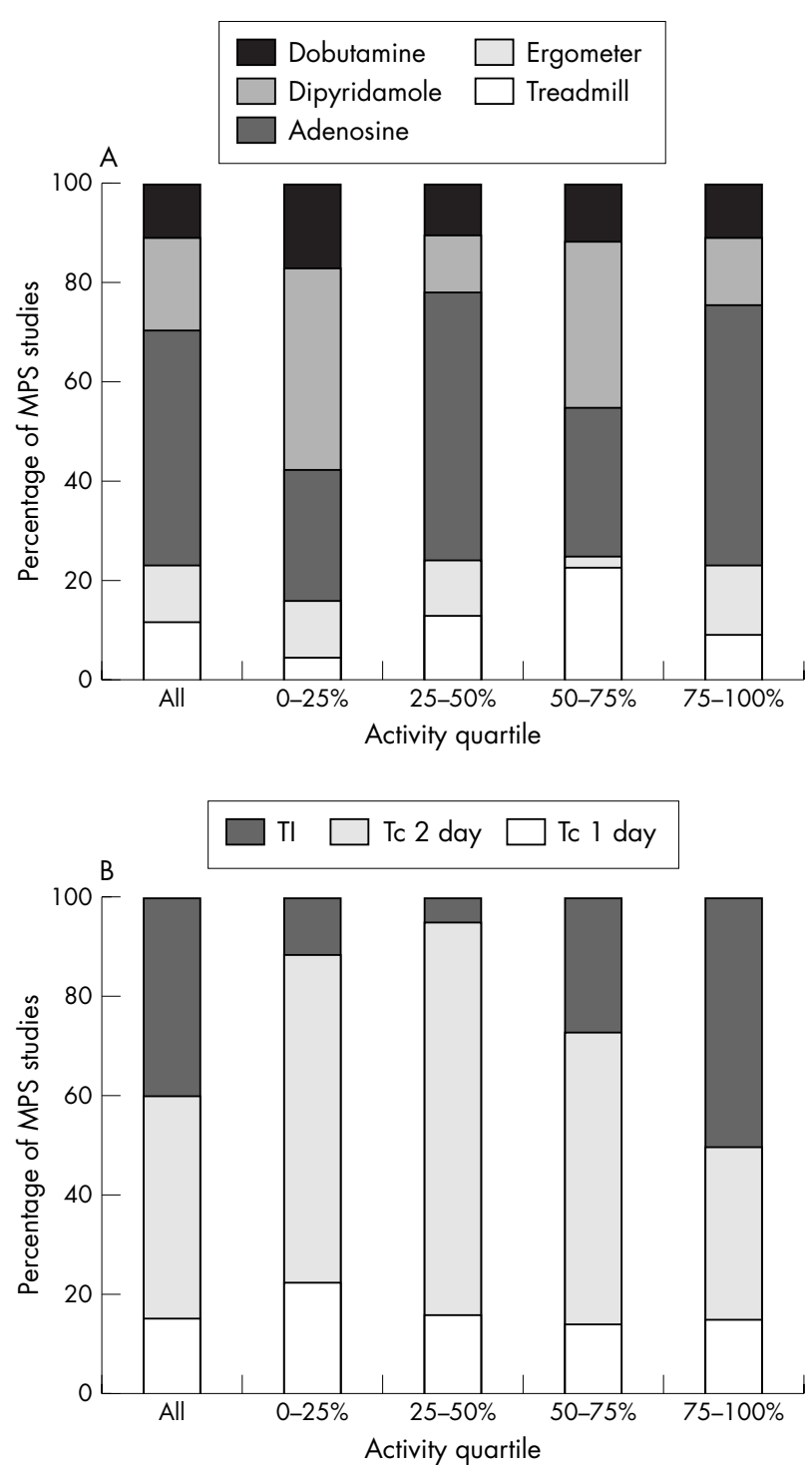

Figure 3 Technical aspects of MPS: comparison between centres in the four quartiles for overall activity. (A) Methods of stress. (B) Radiopharmaceuticals and protocols. Tl, thallium-201, Tc, technetium-99m (sestamibi or tetrofosmin).

quieter centres; $\mathrm{p}=\mathrm{NS}$ ), and $14 \%$ of SPECT MPS studies overall involved attenuation correction.

\section{MPS viability studies}

Four per cent of MPS studies were performed specifically to look for viable and hibernating myocardium. Thallium-201, rather than a technetium-99m based tracer, was used in 59\% of departments in this setting; $77 \%$ of the departments which used a technetium-99m based tracer administered a nitrate before the resting injection.

\section{MPS reporting}

A cardiologist was involved in MPS reporting in $41 \%$ of departments, whether alone (12\%), or in association with a physicist $(20 \%)$, a radiologist $(7 \%)$, or a nuclear physician $(2 \%)$. A radiologist was involved in $37 \%$ of departments (alone $22 \%$, with a physicist $8 \%$, with a cardiologist $7 \%$ ), a nuclear physician in $22 \%$ (alone $20 \%$, with a cardiologist $2 \%$ ), and a physicist in $36 \%$ (alone $8 \%$, with a cardiologist $20 \%$, with a radiologist $8 \%$ ). Overall, a cardiologist was involved in the reporting of $35 \%$ of all MPS studies. Eighty six per cent of
MPI studies were reported from a computer screen rather than from a hard copy ( $94 \%$ for departments in the highest activity quartile, $68 \%$ for quieter centres; $p<0.0001$ ). The raw data were scrutinised before reporting in $73 \%$ of MPS studies $(81 \%$ for busy centres, $56 \%$ for the less busy; $\mathrm{p}<0.0001)$.

\section{DISCUSSION}

\section{National level of MPS activity}

There has been a gradual increase in MPS activity in the UK over the 12 years covered by the BNCS surveys (fig 1). ${ }^{6-8}$ Nevertheless, compared with the rest of western Europe and the USA, the activity level for MPS in the UK remains very low despite one of the highest standardised mortality rates for coronary disease in the world. ${ }^{7}$ In 1994, the European Union average was 2200 MPS studies per million population per year compared with just 560 per million per year in the UK. The rate in the USA in 1993 was more than 10000 per million per year. In 1994 the BCS accepted that greater numbers of MPS studies needed to be performed in the UK, and set a target of 2200 per million per year, the then European average. ${ }^{9}$ It is clear from the 2000 survey that this target has yet to be achieved six years later, and it is likely that since 1994 the European average and hence the target has risen substantially.

The BCS's 1994 target of 2200 MPSs per million per year is conservative in relation to NSF targets set in 2000 for other cardiac procedures. ${ }^{3}$ Professional advice, echoed in the NICE appraisal, is that when MPS is used as the "gatekeeper" to coronary angiography, the ratio of MPS to angiography should be at least 2:1. ${ }^{15}$ However, this survey indicates that in centres able to perform both MPS and coronary angiography, the ratio is only $0.5: 1$. Assuming a $2: 1$ ratio for angiography to revascularisation, the ratio of MPS to revascularisation should be 4:1. The NSF target of 1500 revascularisations per million population per year therefore implies a need for 6000 MPSs per million per year. The recommended activity level for MPS of 4000 per million per year made by the professional societies is therefore conservative, but is nevertheless significantly higher than the current level. ${ }^{1}$

\section{MPS activity at local level}

The survey results suggest a significant lack of MPS capacity in many nuclear medicine departments, and a small number of large departments account for a high proportion of overall national activity. The vast majority of departments possess at least one gamma camera capable of tomographic imaging, and yet only half perform MPS. Only five departments possess a gamma camera dedicated to cardiac imaging. The majority of nuclear medicine departments performing MPS do so on a small scale: the median level of 256 per year is equivalent to a list of six patients once per week.

In their submission to NICE, the UK professional bodies suggested a suitable waiting time for MPS of six weeks for a routine study and one week for an urgent study. ${ }^{15}$ The median waiting time for a routine MPS study in this survey was 16 weeks, which is longer than the NSF target for coronary angiography of 12 weeks. With such a discrepancy, cardiac catheterisation may become a more efficient way of investigating at-risk patients than the more cost effective non-invasive alternative.

There is evidence of a lack of engagement of cardiologists with nuclear cardiology. In all but three centres MPS is performed in general nuclear medicine departments outside the direct experience and supervision of referring cardiologists. This is in stark contrast to other cardiac investigations such as echocardiography and angiography. According to the 2000 survey, a cardiologist is routinely involved in the 
reporting of MPS studies in fewer than half of centres and only $35 \%$ of studies overall. Given the subtleties in the modern treatment of coronary disease, at least some cardiology input is desirable to ensure that reports are not only technically accurate but also management orientated. A previous BNCS audit of 90 MPS studies from 18 UK centres found that while studies were technically adequate in the vast majority of cases, the text of the final report was inadequate in one third of the studies: half were felt to be factually incorrect, and half were essentially correct but misleading because of poor wording. ${ }^{10}$ Until nuclear cardiology services are adequately resourced and cardiologists become more involved, the argument that MPS is clinically valuable may remain largely academic in many hospitals.

\section{Technical issues}

Procedure guidelines on both sides of the Atlantic recommend dynamic exercise as the first line stress technique in MPS, as it provides important prognostic information that is independent of the imaging results. ${ }^{11}{ }^{12}$ Nevertheless, there has been a move away from dynamic exercise in the UK, and more than half of centres now use pharmacological stress exclusively. This may reflect a perception that pharmacological stress can be performed more safely and efficiently in a nuclear medicine department, remote from cardiology staff and expertise. Compared with smaller centres, larger centres are more likely to use adenosine than dipyridamole, and more likely to combine vasodilator stress with submaximal dynamic exercise. These refinements may improve patient throughput by making stress lists more efficient (fewer and shorter lived adverse events) and improving image quality (better gut clearance of technetium-99m agents). ${ }^{13}$ Thallium201 has been displaced as the most common perfusion tracer, and $60 \%$ of studies are now performed using technetium$99 \mathrm{~m}$ based agents. Thallium-201 continues to play a significant role in the larger centres, probably reflecting a combination of familiarity and ease of availability for out-ofhours work (it has a longer half life than technetium-99m, and so can be used on the evening of delivery or the following day).

SPECT imaging is more spatially discriminative and accurate than planar imaging, particularly in patients with relatively mild or single vessel disease, and is recommended in procedure guidelines for MPS. ${ }^{11}{ }^{1214}{ }^{15}$ It is therefore reassuring that only $12 \%$ of studies were still being performed using planar acquisitions; these were concentrated in a handful of centres. Gating of SPECT acquisitions is now well established as a valuable method of identifying attenuation artefacts, and provides important prognostic information about left ventricular function. ${ }^{16}{ }^{17}$ However, only 28\% of centres were doing any gated SPECT, and overall only $22 \%$ of MPS studies involved gating. This was despite the fact that the majority of studies employed a technetium-99m based tracer and were therefore candidates for gating (gating of thallium-201 studies is not feasible in the UK because of the limited dosage permitted). Larger centres were more likely to perform gating than smaller centres, perhaps reflecting the more rapid introduction of novel techniques. Routine attenuation correction is recommended by the American Society of Nuclear Cardiology and the Society of Nuclear Medicine, but increases the complexity of SPECT imaging. ${ }^{18}$ Unsurprisingly, it is not yet widely used in the UK.

Reporting practices were generally good. The raw data were scrutinised in the majority of cases, while reporting was usually done from a computer screen rather than a hard copy. The busiest departments tended to be the most scrupulous, presumably reflecting greater expertise.

\section{Conclusions}

The level of MPS activity in the UK remains low, whether in relation to other cardiac investigations or to practice in comparable industrialised nations. MPS is under resourced in most hospitals, and provided as a low volume service with unacceptably long waiting times. The lack of involvement by cardiologists in MPS and the emphasis on cardiac catheterisation exemplified by the NSF document may further compound the perception that MPS is not a useful investigation in routine use, despite the massive body of evidence to the contrary. The recent NICE appraisal is expected to provide a strong impetus for further development, perhaps not just of MPS but of functional imaging in general. Regular surveys of MPS activity will continue to provide an important vehicle for monitoring the use of resources in the care of patients with known or suspected coronary disease.

\section{Authors' affiliations}

A D Kelion, Harefield Hospital, Harefield, UK

C Anagnostopoulos, Royal Brompton Hospital, London, UK

M Harbinson, Queen's University Belfast, Belfast, UK

R Underwood, Imperial College, London, UK

M Metcalfe, Aberdeen Royal Infirmary, Aberdeen, UK

\section{REFERENCES}

1 Underwood SR, Anagnostopoulos C, Cerqueira M, et al. Myocardial perfusion scintigraphy: the evidence. Eur $J$ Nucl Med Mol Imaging 2004;31:261-91.

2 De Bono D. Investigation and management of stable angina: revised guidelines 1998. Heart 1999;81:546-58.

3 Department of Health. National service framework for coronary heart disease. London: Department of Health, 2000.

4 Wong $Y$, Rodwell A, Dawkins S, et al. Sex differences in investigation results and treatment in subjects referred for investigation of chest pain. Heart $2001 ; 85: 149-52$.

5 National Institute for Health and Clinical Excellence. Technology appraisal guidance 73. Myocardial perfusion scintigraphy for the diagnosis and management of angina and myocardial infarction. London: NICE, 2003.

6 Underwood R, Gibson C, Tweddel A, et al. A survey of nuclear cardiological practice in Great Britain. The British Nuclear Cardiology Group. Br Heart J 1992;67:273-7.

7 Pennell DJ, Prvulovich E, Tweddel A, et al. Nuclear cardiology in the UK: British Nuclear Cardiology Society survey 1994. Nud Med Comm 1998;19:305-13.

8 Prvulovich E, Metcalfe MJ. Nuclear cardiology in the UK: activity and practice 1997. Eur J Nucl Med 2002;29:553-8.

9 British Cardiac Society. A report of a working group of the British Cardiac Society. Cardiology in the district general hospital. Br Heart J 1994;72:303-8.

10 Prvulovich EM, Jarritt PH, Vivian GC, et al. Quality assurance in myocardial perfusion tomography: a collaborative BNCS/BNMS audit programme. Nucl Med Comm 1998;19:831-8.

11 DePuey EG, Garcia E, Borges-Neto S. Updated imaging guidelines for nuclear cardiology procedures, part 1. J Nucl Cardiol 2001;8:G1-58.

12 Anagnostopoulos C, Harbinson M, Kelion A, et al. Procedure guidelines for radionuclide myocardial perfusion imaging. Heart 2004;90(suppl I):i1-10.

13 Pennell DJ, Mavrogeni SI, Forbat SM, et al. Adenosine combined with dynamic exercise for myocardial perfusion imaging. J Am Coll Cardiol 1995;6:1300-9.

14 Fintel DJ, Links JM, Brinker JA, et al. Improved diagnostic performance of exercise thallium-201 single photon emission computed tomography over planar imaging in the diagnosis of coronary artery disease: a receiver operating characteristic analysis. J Am Coll Cardiol 1989;13:600-12.

15 Hacot JP, Bojovic M, Delonca J, et al. Comparison of planar imaging and single-photon emission computed tomography for the detection and localization of coronary artery disease. Int J Card Imaging 1993;9:113-9.

16 DePuey EG, Rozanski A. Using gated technetium-99m-sestamibi SPECT to characterize fixed myocardial defects as infarct or artifact. J Nucl Med 1995;36:952-5.

17 Sharir T, Germano G, Kavanagh PB, et al. Incremental prognostic value of post-stress left ventricular ejection fraction and volume by gated myocardial perfusion single photon emission computed tomography. Circulation 1999; 100:1035-42.

18 American Society of Nuclear Cardiology, Society of Nuclear Medicine. Attenuation correction of myocardial perfusion SPECT scintigraphy: a joint position statement by the American Society of Nuclear Cardiology and the Society of Nuclear Medicine. www.asnc.org. 